\title{
Preconception care in mental health services: planning for a better future
}

Raquel Catalao, Sue Mann, Claire Wilson and Louise M. Howard

\section{Summary}

It is increasingly recognised that the preconception period is a window of opportunity to intervene to improve outcomes for women and the next generation. The importance of preconception mental health and comorbidity problems has not traditionally been taken into account by policy makers or mental health service providers. We argue that by addressing preconception physical and mental health in men and women, medical health professionals could improve health outcomes across the whole life course.

\section{Declaration of interest}

None.

\section{Keywords}

Preconception; perinatal psychiatry; comorbidity; policy; pregnancy planning.

\section{Copyright and usage}

(c) The Royal College of Psychiatrists 2019
Raquel Catalao (pictured) is a National Institute for Health Research (NIHR) Academic Clinical Fellow in Psychiatry at South London and Maudsley NHS Foundation Trust and in the Section of Women's Mental Health, Institute of Psychiatry, Psychology and Neuroscience, King's College London. Sue Mann is a Public Health Consultant and Medical Adviser in Reproductive Health Programmes and Priorities at Public Health England. Claire Wilson is a Medical Research Council Clinical Research Training Fellow in the Section of Women's Mental Health, Institute of Psychiatry, Psychology and Neuroscience, King's College London. Louise M. Howard is a NIHR Professor in Women's Mental Health in the Section of Women's Mental Health, Institute of Psychiatry, Psychology and Neuroscience, King's College London, and a NIHR Senior Investigator and consultant Psychiatrist at South London and Maudsley NHS Foundation Trust.

The increasing recognition of the burden of perinatal mental disorders and their wide-ranging effects on maternal and children's health has led to the development of policy initiatives aimed at transforming perinatal mental healthcare in the UK, including specialist community perinatal services for women with severe or complex conditions. However, mounting evidence suggests that perinatal mental disorders are often preceded by mental health problems that begin before pregnancy, including in adolescence or young adulthood, and these maternal preconception disorders are associated with adverse effects for mother, infant and child, including obstetric complications, poorer mother-infant bonding and infant emotional reactivity (a marker of vulnerability to subsequent mental health problems). ${ }^{1}$ Severe and common mental health disorders in women have also been shown to be associated with higher rates contraceptive non-use, misuse or less effective method choice, as well as teenage and unintended pregnancies. ${ }^{2}$ Although much of the preconception and perinatal literature has focused on mothers, there is also some emerging evidence for the role of the father's preconception mental health in shaping offspring outcomes. Mechanisms potentially include epigenetic changes in paternal and maternal gametes as well as the early embryo in response to a suboptimal nutritional environment, substance use and stress.

A recent Lancet series on preconception health proposed a preconception conceptual framework that includes a biological perspective (the days to weeks before embryo development), an individual perspective (a conscious intention to conceive) and a public health perspective (months or years beforehand) to address preconception risk factors such as diet and obesity. Mental health should also be recognised as an important preconception health indicator and be included in public health interventions aimed at this period. ${ }^{3}$ A multimorbidity life-course approach to preconception care would enable health behaviours and risk factors to be addressed before there is explicit intention to conceive as well as optimising health in a way that would benefit a pregnancy were it to occur.

Although preconception health has traditionally not been a focus of mental health services, the new perinatal community mental health services being rolled out in England will be offering preconception counselling to all women referred with moderate to severe mental illness who are planning a pregnancy. In addition, with recent Medicines and Healthcare Products Regulatory Agency guidance ${ }^{4}$ stating that valproate must no longer be used in any woman of reproductive age unless she has a Pregnancy Prevention Programme in place, mental health consultations such as medication reviews are an ideal opportunity for a discussion around pregnancy planning, to optimise contraception where appropriate and to advise women of reproductive age on wider aspects of preconception care, such as nutrition and weight management, smoking, alcohol and other substances and folic acid supplementation. This could occur across services, including early intervention services, generic community and in-patient services and primary care. There is also an opportunity to integrate a pregnancy planning and pregnancy prevention consultation with regular physical health checks for people with severe mental illness, alongside the recommended lifestyle interventions focused on diet and physical activity. An important target for intervention is smoking, which is particularly prevalent among people with mental disorder and is the single biggest modifiable risk factor for poor birth outcomes. Health promotion messages can include wider determinants of health, such as sleep, nutrition, social inclusion and support for stress-relieving activities such as exercise and mindfulness. However, there is evidence that people with severe mental illness may not respond to traditional public health campaigns and therefore targeted interventions in appropriate settings may be key to change outcomes in this group. ${ }^{5}$ Mental health professionals should also regularly ask about domestic violence and abuse and sensitively respond to disclosure. Thinking about preconception mental health early can help professionals and potential parents to minimise safeguarding concerns in the perinatal period and beyond; men and women can be advised on how to plan for a child, which is a central part of recovery, particularly for women, although often neglected by services. 
Raising awareness among staff and providing training tools and resources will be crucial; it will require individual provider behaviour change that could draw on the Making Every Contact Count model (https://www.makingeverycontactcount.co.uk/) that has been used in other settings; furthermore, sustainable change will likely require a whole systems approach where collaborative models with other local services with agreed referral pathways ensure clear communication among professionals and the delivery of interventions. This is particularly important for our population who may not access other parts of the health service and is particularly vulnerable to multimorbidity. Primary healthcare and secondary healthcare services offering preconception advice should be aware of the burden of perinatal mental health disorders and feel equipped to signpost high-risk women, such as those with a personal history of bipolar affective disorder, ${ }^{6}$ to appropriate mental health services. In addition, mental health relevant preconception counselling should not be restricted to women with severe mental illness and should be considered for all women with vulnerability factors including those who experienced adverse childhood experiences or trauma, or have personality difficulties, as they can find the perinatal period particularly challenging. Similarly, preconception counselling by perinatal mental health teams should be able to elicit important physical health comorbidities and refer accordingly.

National Institute for Health and Care Excellence guidance ${ }^{7}$ already recommends that all women of childbearing potential who have a new, existing or past mental health problem should be offered a discussion on pregnancy planning. However, in view of increasing evidence on how preconception mental health affects the next generation, mental health services need to consider how to best normalise preconception care delivery for all women and men of reproductive age. Thus, it is time for mental health services to recognise not only each individual's history, but also their future as a potential parent, and play an active role in promoting healthier behaviours, with potential benefits for the health and longevity of our patients and their future children.

Raquel Catalao (D), NIHR Academic Clinical Fellow in Psychiatry, South London and Maudsley NHS Foundation Trust and Section of Women's Mental Health, Institute of Psychiatry, Psychology and Neuroscience, King's College London, UK; Sue Mann, Public Health Consultant and Medical Adviser in Reproductive Health Programmes and
Priorities, Public Health England, UK; Claire Wilson, Medical Research Council Clinical Research Training Fellow, Section of Women's Mental Health, Institute of Psychiatry, Psychology and Neuroscience, King's College London, UK; Louise M. Howard (D), NIHR Professor in Women's Mental Health, Section of Women's Mental Health, Institute of Psychiatry, Psychology and Neuroscience, King's College London, UK; and NIHR Senior Investigator and Consultant Psychiatrist, South London and Maudsley NHS Foundation Trust, UK

Correspondence: Raquel Catalao, Section of Women's Mental Health, Institute of Psychiatry, Psychology and Neuroscience, King's College London, Dr Crespigny Park, London SE5 8AF, UK. Email: raquel.catalao@kcl.ac.uk

First received 20 Jun 2019, final revision 13 Aug 2019, accepted 23 Aug 2019

\section{Acknowledgements}

The views expressed are those of the authors and not necessarily those of the NHS, the NIHR, Public Health England or the MRC.

\section{References}

1 Spry E, Moreno-Betancur M, Becker D, Romaniuk H, Carlin J, Molyneaux E, et al. Maternal mental health and infant emotional reactivity: a 20-year two-cohort study of preconception and perinatal exposures. Psychol Med Apr 2019; in press.

2 Matevosyan NR. Reproductive health in women with serious mental illnesses: a review. Sex Disabil 2009; 27(2): 109-18.

3 Wilson C, Howard LM, Reynolds RM, Simonoff E, Ismail K. Preconception health. Lancet 2018; 392(10161): 2266-7.

4 Medicines and Healthcare Products Regulatory Agency. Drug Safety Update. Valproate Medicines (Epilim $\mathbf{v}$, Depakote $\mathbf{v}$ ): Contraindicated in Women and Girls of Childbearing Potential unless Conditions of Pregnancy Prevention Programme are Met. Medicines and Healthcare Products Regulatory Agency, 2018 (https://www.gov.uk/drug-safety-update/valproate-medicines-epilimdepakote-contraindicated-in-women-and-girls-of-childbearing-potentialunless-conditions-of-pregnancy-prevention-programme-are-met).

5 Gilbody S, Peckham E, Bailey D, Arundel C, Heron P, Crosland S, et al. Smoking cessation for people with severe mental illness (SCIMITAR+): a pragmatic randomised controlled trial. Lancet Psychiatry 2019; 6(5): 379-90.

6 Dolman C, Jones IR, Howard LM. Women with bipolar disorder and pregnancy: factors influencing their decision-making. BJPsych Open 2016; 2(5): 294-300.

7 National Institute for Health and Care Excellence. Antenatal and Postnatal Mental Health: Clinical Management and Service Guidance. Clinical Guideline [CG192]. NICE, 2018 (https://www.nice.org.uk/guidance/cg192). 